\title{
GENERALIZED SCALAR OPERATORS AS DILATIONS
}

\author{
HARI BERCOVICI AND SRDJAN PETROVIĆ
}

(Communicated by Palle E. T. Jorgensen)

\begin{abstract}
It is shown that polynomially bounded operators on Banach spaces have polynomially bounded dilations which have spectrum in the unit circle and are generalized scalar. The proof also yields a description of all compressions of generalized scalar operators with spectrum in the unit circle.
\end{abstract}

\section{INTRODUCTION}

Let $\mathscr{H}$ denote a complex Hilbert space, and let $\mathscr{L}(\mathscr{H})$ denote the algebra of all bounded linear operators on $\mathscr{H}$. Recall that an operator $S$ in $\mathscr{L}(\mathscr{H})$ is said to be polynomially bounded if there exists an $M \geq 1$ such that

$$
\|p(S)\| \leq M \sup \{|p(\zeta)|:|\zeta|=1\}
$$

for all polynomials $p$. Halmos [2] raised the question whether every polynomially bounded operator is similar to a contraction (i.e., an operator of norm at most 1); this question is still open. Now, a famous theorem of Sz.-Nagy [5] states that every contraction has a unitary dilation, i.e., if $S$ is a contraction in $\mathscr{L}(\mathscr{H})$, then there exists a Hilbert space $\mathscr{K}$ containing $\mathscr{H}$ and a unitary operator $T \in \mathscr{L}(\mathscr{K})$ such that $S^{n}=P T^{n} \mid \mathscr{H}$ for every $n=0,1,2, \ldots$, where $P$ is the orthogonal projection in $\mathscr{L}(\mathscr{K})$ whose range is $\mathscr{H}$. Sz.-Nagy's result implies that any operator similar to a contraction has a dilation which is similar to a unitary operator. This suggests that one way to approach Halmos's question would be to look at dilations of polynomially bounded operators which have interesting additional properties. In fact, it was shown in [3] and [4] that every polynomially bounded operator has a polynomially bounded dilation which

(1) has spectrum contained in the unit circle,

(2) is generalized scalar in the sense of [1], and

(3) is weakly centered.

In this paper we show that a version of the construction in [4] provides a similar result for polynomially bounded operators on arbitrary Banach space. In fact, this construction provides a characterization of all operators which are compressions (in a sense to be defined below) of arbitrary generalized scalar operators with spectrum on the unit circle. Let us note however that in the case of Hilbert space our construction does not yield weakly centered dilations.

Received by the editors November 12, 1993.

1991 Mathematics Subject Classification. Primary 47A20. 
A form of our construction was known to Ciprian Foias in the 1960s (unpublished). Namely, he observed that a doubly infinite matrix of the form

$$
\left(\begin{array}{llllll}
\ddots & & & & & \\
& I & & & & \\
& & I & & & \\
& S & I & & \\
& & & I & \\
& & & & & \ddots
\end{array}\right)
$$

is generalized scalar if and only if $\left\|S^{n}\right\|$ grows polynomially as $n \rightarrow \infty$.

\section{THE MAIN ReSULT}

Let us recall that an operator $T$ on a Banach space $\mathscr{Z}$ with $\sigma(T) \subset \mathbb{T}=\{\lambda \in$ $\mathbb{C}:|\lambda|=1\}$ is a generalized scalar operator if it has a continuous functional calculus with $C^{\infty}(\mathbb{T})$ functions. It is shown [1] that this condition is equivalent to a growth condition of the form

$$
\left\|T^{n}\right\|=O\left(|n|^{\alpha}\right), \quad n \in \mathbb{Z}, \quad|n| \rightarrow \infty,
$$

with $\alpha \geq 0$.

Assume now that $T$ acts on $\mathscr{Z}$, and $\mathscr{M}, \mathscr{N}$ are two closed invariant subspaces for $T$ such that $\mathscr{M} \subset \mathscr{N}$. Then one can define an operator $S \in$ $\mathscr{L}(\mathscr{N} / \mathscr{M})$ by the formula

$$
S(x+\mathscr{M})=T x+\mathscr{M}, \quad x \in \mathscr{N} .
$$

The operator $S$ will be called a compression of $T$. Now, if $S$ is a compression of $T$, and $T$ satisfies (1), then clearly

$$
\left\|S^{n}\right\|=O\left(n^{\alpha}\right), \quad n \in \mathbb{N}, \quad|n| \rightarrow \infty .
$$

Our main result is a converse of this assertion. More precisely, we have

Theorem 2.1. Suppose that $\alpha \geq 0$, and let $S \in \mathscr{L}(\mathscr{Z})$ satisfy (2). Then there exists a Banach space $\mathscr{K}$ and an operator $T \in \mathscr{L}(\mathscr{K})$ such that :

(a) $S$ is a compression of $T$.

(b)

$$
\left\|T^{n}\right\|= \begin{cases}O\left(|n|^{\frac{\alpha+1}{2}}\right) & \text { if } \alpha<1, \\ O(|n| \log |n|) & \text { if } \alpha=1, \\ O\left(|n|^{\alpha}\right) & \text { if } \alpha \geq 1\end{cases}
$$

for $n \in \mathbb{Z},|n| \rightarrow \infty$. In particular, $T$ is generalized scalar.

(c) $\sigma(T)=\mathbb{T}$.

If $S$ is polynomially bounded, then $T$ can be chosen to be polynomially bounded as well.

Proof. We take $\mathscr{K}=\ell^{2}(\mathscr{X})$. In other words, $\mathscr{K}$ is a two-way infinite direct sum of infinitely many copies of $\mathscr{Z}$, and if $x=\left(x_{n}\right)_{n \in \mathbf{Z}} \in \mathscr{K}$, then $\|x\|^{2}=$ 
$\sum_{-\infty}^{\infty}\left\|x_{n}\right\|^{2}$. We define $T$ to be a two-way infinite matrix acting on $\mathscr{K}$ as

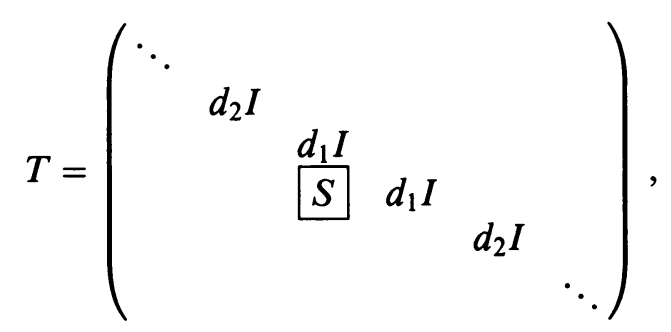

where the omitted entries are understood to be zero operators, the boxed entry denotes $(0,0)$ position, $I$ denotes the identity operator on $\mathscr{X}$, and

$$
d_{i}=\left(\frac{i}{i+1}\right)^{\frac{\alpha+1}{4}}, \quad i \in \mathbb{N}
$$

The assertion (a) is trivial, and the assertion (c) will clearly follow from (b) by computing the spectral radii of $T$ and $T^{-1}$. In fact it is not hard to see that $\sigma(T)=\mathbb{T}$, but we do not need this fact. We concentrate first on assertion (b).

First, we denote $\mathscr{M}=\underset{0}{\oplus} \mathscr{X}_{i}$, where $\mathscr{X}_{i}=\mathscr{X}$, and

$$
R=\left(\begin{array}{llll}
S & d_{1} I & & \\
& & d_{2} I & \\
& & & \ddots .
\end{array}\right)
$$

For every $n \in \mathbb{N}$ we have

$$
R^{n}=\left(\begin{array}{lllllll}
S^{n} & d_{1} S^{n-1} & \ldots & d_{1} d_{2} \ldots d_{n-1} S & d_{1} \ldots d_{n} & & \\
& & & & & d_{2} d_{3} \ldots d_{n+1} & \\
& & & & & \ddots
\end{array}\right) .
$$

Let $x=\left(x_{0}, x_{1}, \ldots\right) \in \mathscr{M}$ be an arbitrary vector of norm 1 . Then

$$
\left\|R^{n} x\right\|=\left\|\left(\begin{array}{c}
S^{n} x_{0}+d_{1} S^{n-1} x_{1}+\cdots+d_{1} d_{2} \ldots d_{n} x_{n} \\
d_{2} d_{3} \ldots d_{n+1} x_{n+1} \\
\vdots
\end{array}\right)\right\|
$$

so that

$$
\begin{aligned}
\left\|R^{n} x\right\|^{2}= & \left\|S^{n} x_{0}+d_{1} S^{n-1} x_{1}+\cdots+d_{1} \ldots d_{n} x_{n}\right\|^{2}+\left\|d_{2} \ldots d_{n+1} x_{n+1}\right\|^{2}+\cdots \\
\leq & \left(\left\|S^{n}\right\|\left\|x_{0}\right\|+\left\|d_{1} S^{n-1}\right\|\left\|x_{1}\right\|+\cdots+d_{1} \ldots d_{n}\left\|x_{n}\right\|\right)^{2} \\
& +\left(d_{2} \ldots d_{n+1}\right)^{2}\left\|x_{n+1}\right\|^{2}+\cdots \\
\leq & \left(\left\|S^{n}\right\|^{2}+\left\|d_{1} S^{n-1}\right\|^{2}+\cdots+\left(d_{1} \ldots d_{n}\right)^{2}\right)\left(\left\|x_{0}\right\|^{2}+\cdots+\left\|x_{n}\right\|^{2}\right) \\
& +\left\|x_{n+1}\right\|^{2}+\left\|x_{n+2}\right\|^{2}+\cdots .
\end{aligned}
$$

We conclude that $\left\|R^{n}\right\|^{2} \leq 1+\left\|S^{n}\right\|^{2}+\left\|d_{1} S^{n-1}\right\|^{2}+\cdots+\left(d_{1} \ldots d_{n}\right)^{2}$. Thus $\left\|R^{n}\right\|^{2}$ grows no faster than

$$
1+n^{2 \alpha}+(n-1)^{2 \alpha} \frac{1}{2^{\frac{\alpha+1}{2}}}+(n-2)^{2 \alpha} \frac{1}{3^{\frac{\alpha+1}{2}}}+\cdots+2^{2 \alpha} \frac{1}{(n-1)^{\frac{a+1}{2}}}+\frac{1}{n^{\frac{\alpha+1}{2}}}+\frac{1}{(n+1)^{\frac{\alpha+1}{2}}}
$$


which is dominated by

$$
n^{2 \alpha} \sum_{k=1}^{n+1} \frac{1}{k^{\frac{\alpha+1}{2}}} .
$$

We notice that if $\alpha<1$, the last expression is of the order of growth $n^{2 \alpha} \cdot n^{\frac{1-\alpha}{2}}=$ $n^{\frac{1+3 \alpha}{2}}$, and therefore $\left\|R^{n}\right\|=O\left(n^{\frac{1+3 \alpha}{4}}\right)$. If $\alpha>1$, the sum in (4) is dominated by a convergent series, so $\left\|R^{n}\right\|=O\left(n^{\alpha}\right)$. If $\alpha=1$, the sum in (4) has the order of growth of $\log n$, and therefore $\left\|R^{n}\right\|=O(n \sqrt{\log n})$.

Next we notice that the matrix (3) can be rewritten as

$$
T=\left(\begin{array}{ccc}
\ddots & & \\
& d_{2} I & \\
& & d_{1} P \\
& & R
\end{array}\right)
$$

relative to $\mathscr{K}=\left(\underset{-\infty}{-1} \mathscr{X}_{i}\right) \oplus \mathscr{M}$, where $\mathscr{X}_{i}=\mathscr{X}$ and $P: \mathscr{M}=\bigoplus_{i=0}^{\infty} \mathscr{X}_{i} \rightarrow \mathscr{X}_{-1}$ is given by $P\left(x_{0}, x_{1}, \ldots\right)=x_{0}$. Of course, $T$ is unitarily equivalent to the matrix

$$
\left(\begin{array}{ccc}
R & & \\
d_{1} P & & \\
& d_{2} I & \\
& & \ddots .
\end{array}\right)
$$

and we shall identify them. Thus

$$
T^{n}=\left(\begin{array}{ccc}
R^{n} & & \\
d_{1} P R^{n-1} & & \\
\vdots & & \\
d_{n-1} \ldots d_{1} P R & & \\
d_{n} \ldots d_{1} P & & \\
& d_{n+1} \ldots d_{2} I & \\
& & \ddots
\end{array}\right) .
$$

Let $y=\left(y_{0}, y_{1}, \ldots\right) \in \mathscr{K}$ be an arbitrary vector of norm 1 . Then

$$
\begin{aligned}
& \left\|T^{n} y\right\|^{2}=\left\|\left(\begin{array}{c}
R^{n} y_{0} \\
d_{1} P R^{n-1} y_{0} \\
\vdots \\
d_{n-1} \ldots d_{1} P R y_{0} \\
d_{n} \ldots \tilde{d}_{1} y_{0} \\
d_{n+1} \ldots d_{2} y_{1} \\
\vdots \\
\vdots
\end{array}\right)\right\|^{2} \\
& =\left\|R^{n} y_{0}\right\|^{2}+\left\|d_{1} P R^{n-1} y_{0}\right\|^{2}+\cdots+\left\|d_{n} \ldots d_{1} P y_{0}\right\|^{2}+\left\|d_{n+1} \ldots d_{2} y_{1}\right\|^{2}+\ldots \\
& \leq\left(\left\|R^{n}\right\|^{2}+d_{1}{ }^{2}\left\|R^{n-1}\right\|^{2}+\cdots+\left(d_{n} \ldots d_{2} d_{1}\right)^{2}\right)\left\|y_{0}\right\|^{2}+\left(d_{n+1} \ldots d_{2}\right)^{2}\left\|y_{1}\right\|^{2}+\ldots \\
& \leq\left(\left\|R^{n}\right\|^{2}+d_{1}{ }^{2}\left\|R^{n-1}\right\|^{2}+\cdots+\left(d_{n} \ldots d_{2} d_{1}\right)^{2}\right)\left\|y_{0}\right\|^{2}+\left\|y_{1}\right\|^{2}+\left\|y_{2}\right\|^{2}+\ldots .
\end{aligned}
$$


We conclude that

$$
\left\|T^{n}\right\|^{2} \leq 1+\left\|R^{n}\right\|^{2}+d_{1}^{2}\left\|R^{n-1}\right\|^{2}+\cdots+\left(d_{n} \ldots d_{2} d_{1}\right)^{2} .
$$

Again we consider separately the cases $\alpha<1, \alpha>1$, and $\alpha=1$. If $\alpha<1$, then $\left\|T^{n}\right\|^{2}$ obviously grows no faster than

$$
1+n^{\frac{1+3 \alpha}{2}}+(n-1)^{\frac{1+3 \alpha}{2}} \frac{1}{2^{\frac{1+\alpha}{2}}}+(n-2)^{\frac{1+3 \alpha}{2}} \frac{1}{3^{\frac{1+\alpha}{2}}}+\cdots+\frac{1}{n^{\frac{1+\alpha}{2}}}+\frac{1}{(n+1)^{\frac{1+\alpha}{2}}} .
$$

Since this sum is dominated by $n^{\frac{1+3 \alpha}{2}} \sum_{k=1}^{n+1} \frac{1}{k^{\frac{1+\alpha}{2}}}$, we obtain in this case that

$$
\left\|T^{n}\right\|=O\left(n^{\frac{1+\alpha}{2}}\right), \quad \text { for } n=0,1,2, \ldots
$$

If $\alpha>1$, then the order of growth of $\left\|T^{n}\right\|^{2}$ is clearly dominated by the order of growth of

$$
1+n^{2 \alpha}+(n-1)^{2 \alpha} \frac{1}{2^{\frac{1+\alpha}{2}}}+(n-2)^{2 \alpha} \frac{1}{3^{\frac{1+\alpha}{2}}}+\cdots+\frac{1}{n^{\frac{1+\alpha}{2}}}+\frac{1}{(n+1)^{\frac{1+\alpha}{2}}},
$$

which is in its turn dominated by $n^{2 \alpha} \sum_{k=1}^{n+1} \frac{1}{k^{\frac{1+\alpha}{2}}}$. Of course, the sum in the last expression is uniformly bounded, so $\left\|T^{n}\right\|=O\left(n^{\alpha}\right)$. Finally, if $\alpha=1,\left\|T^{n}\right\|^{2}$ grows no faster than

$$
1+n^{2} \log n+(n-1)^{2} \log (n-1) \frac{1}{2^{\frac{1+\alpha}{2}}}+\cdots+\frac{1}{n^{\frac{1+\alpha}{2}}}+\frac{1}{(n+1)^{\frac{1+\alpha}{2}}}
$$

which is dominated by $n^{2} \log n \sum_{k=1}^{n-1} \frac{1}{k}$, and since the last sum grows as $\log n$, we obtain that $\left\|T^{n}\right\|=O(n \log n)$.

Next we estimate $\left\|T^{-n}\right\|$ for $n=1,2, \ldots$. An easy calculation shows that

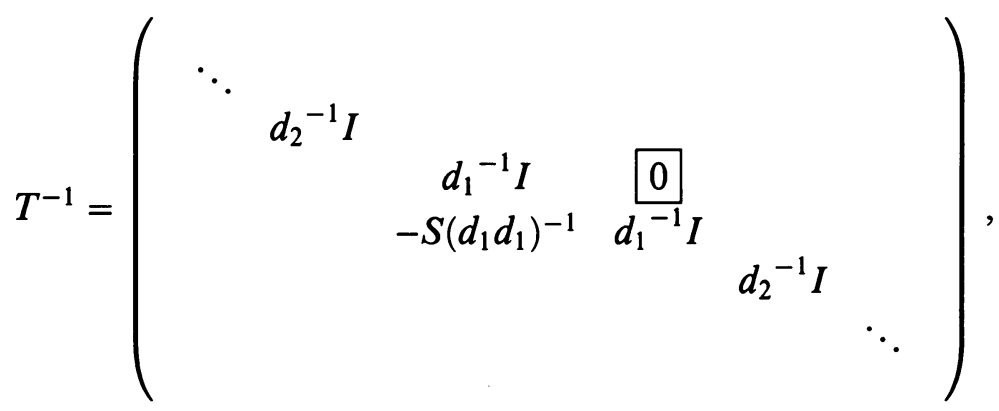


and another calculation yields

$$
T^{-n}=\left(\begin{array}{cccccccc}
\ddots & & & & & & & \\
& \frac{I}{d_{n+1} \ldots d_{2}} & & & & & & \\
& 0 & \frac{I}{d_{n} \ldots d_{1}} & 0 & \ldots & 0 & 0 & \\
& & \frac{-S}{d_{n} \ldots d_{1} d_{1}} & \frac{I}{d_{n-1} \ldots d_{1} d_{1}} & \ddots & & 0 & \\
& & & \ddots & \ddots & \ddots & \vdots & \\
& & & \ddots & \frac{I}{d_{1} d_{1} \ldots d_{n-1}} & 0 & \\
& & & & \frac{d_{1} d_{1} \ldots d_{n}}{d_{1} \ldots d_{n}} & \\
& & & & & 0 & \ddots \\
& & & & & & \ddots
\end{array}\right)
$$

and thus

$$
\begin{gathered}
\left\|T^{-n}\right\| \leq \sup \left\{\ldots,\left(d_{n+1} \ldots d_{2}\right)^{-1},\left(d_{n}, \ldots d_{1}\right)^{-1},\left(d_{n-1} \ldots d_{1} d_{1}\right)^{-1}, \ldots\right. \\
\left.\ldots,\left(d_{1} d_{1} \ldots d_{n-1}\right)^{-1},\left(d_{1} \ldots d_{n}\right)^{-1}, \ldots\right\} \\
\quad+\sup \left\{\left\|S\left(d_{n} \ldots d_{1} d_{1}\right)^{-1}\right\|,\left\|S\left(d_{n-1} \ldots d_{1} d_{1} d_{2}\right)^{-1}\right\|, \ldots,\left\|S\left(d_{1} d_{1} \ldots d_{n}\right)^{-1}\right\|\right\}
\end{gathered}
$$

Since the sequence $\left\{d_{n}^{-1}\right\}$ is bounded, the rate of growth of $\left\|T^{-n}\right\|$ will be determined by the first summand, and since the mentioned sequence is decreasing, the supremum is attained at the term of the form $\left(d_{s} d_{s-1} \ldots d_{1} d_{1} d_{2} \ldots d_{n-s}\right)^{-1}$ which equals

$$
(s+1)^{\frac{1+\alpha}{4}}(n-s+1)^{\frac{1+\alpha}{4}} .
$$

The maximum value of $(x+1)(n-x+1)$ for $x \in[0, n]$ is $(1+n / 2)^{2}$ and therefore $\left\|T^{-n}\right\|=O\left(n^{\frac{1+a}{2}}\right)$. Together with (5) this completes the proof of assertion (b).

Finally, assume that $S$ is polynomially bounded. Once again, we define $T$ as (3) with

$$
d_{i}=\left(\frac{i}{i+1}\right)^{\gamma}
$$

where $\gamma>1 / 2$. Clearly, it suffices to show that in this case $R$ is polynomially bounded. Writing

$$
R=\left(\begin{array}{cc}
S & d_{1} P \\
0 & W
\end{array}\right)
$$

where $W$ is the unilateral backward weighted shift with weight sequence $\left\{d_{i}\right\}_{i \geq 2}$, we see that for a polynomial $p(z)=\sum_{k=0}^{n} a_{k} z^{k}$,

$$
p(R)=\left(\begin{array}{cc}
p(S) & G \\
0 & p(W)
\end{array}\right)
$$

where $G=\sum_{k=1}^{n} a_{k} \sum_{i=0}^{k-1} S^{k-i-1} d_{1} P W^{i}$. Since $S$ is polynomially bounded and $W$ is a contraction, it suffices to show that $\|G\| \leq M\|p\|_{\infty}$ for some $M \geq 1$ 
independent of $p$. Here we have denoted by $\|\cdot\|_{\infty}$ the supremum norm over the unit circle. Let $\left(x_{2}, x_{3}, \ldots\right)$ be an arbitrary vector of norm 1 in $\underset{i=1}{\oplus} \mathscr{X}_{i}$. Then

$$
\begin{aligned}
G\left(x_{2}, x_{3}, \ldots\right) & =\sum_{k=1}^{n} a_{k} \sum_{i=0}^{k-1} S^{k-i-1} d_{1} P W^{i}\left(x_{2}, x_{3}, \ldots\right) \\
& =\sum_{k=1}^{n} a_{k} \sum_{i=0}^{k-1} S^{k-i-1} d_{1} \ldots d_{i+1} x_{i+2} \\
& =\sum_{k=1}^{n} a_{k} \sum_{i=0}^{k-1} S^{k-i-1} \frac{1}{(i+2)^{\gamma}} x_{i+2} \\
& =\sum_{i=0}^{n-1} \sum_{k=i+1}^{n} a_{k} S^{k-i-1} \frac{x_{i+2}}{(i+2)^{\gamma}} \\
& =\sum_{i=0}^{n-1} \frac{1}{(i+2)^{\gamma}}\left(a_{i+1}+a_{i+2} S+\cdots+a_{n} S^{n-i-1}\right) x_{i+2} .
\end{aligned}
$$

Therefore,

$$
\begin{aligned}
\left\|G\left(x_{2}, \ldots\right)\right\| & \leq \sum_{i=0}^{n-1} \frac{1}{(i+2)^{\gamma}}\left\|\left(a_{i+1}+\cdots+a_{m} S^{n-i-1}\right)\right\|\left\|x_{i+2}\right\| \\
& \leq\left(\sum_{i=0}^{n-1} \frac{\left\|\left(a_{i+1}+\cdots+a_{n} S^{n-i-1}\right)\right\|^{2}}{(i+2)^{2 \gamma}}\right)^{1 / 2}\left(\sum_{i=0}^{n-1}\left\|x_{i+2}\right\|^{2}\right)^{1 / 2} .
\end{aligned}
$$

Using the notation $p_{(i)}(z)=a_{i}+a_{i+1} z+\cdots+a_{n} z^{n-i}$ and the fact that $S$ is polynomially bounded and that $\left\|\left(x_{2}, x_{3}, \ldots\right)\right\|=1$ we obtain that

$$
\left\|G\left(x_{2}, \ldots\right)\right\| \leq\left(\sum_{i=0}^{n-1} \frac{M^{2}\left\|p_{(i)}\right\|_{\infty}^{2}}{(i+2)^{2 \gamma}}\right)^{1 / 2} .
$$

It is well known that $\left\|p_{(i)}\right\|_{\infty}$ grows no faster than $\|p\|_{\infty} \log i$, so the proof follows from the convergence of the infinite series $\sum_{i=0}^{\infty} \frac{\log ^{2} i}{(i+2)^{2 \gamma}}$, which is obvious in view of the fact that $\gamma>1 / 2$.

\section{REFERENCES}

1. I. Colojoară and C. Foias, Theory of generalized spectral operators, Gordon and Breach, New York, 1968.

2. P. R. Halmos, Ten problems in Hilbert space, Bull. Amer. Math. Soc. 76 (1970), 887-933.

3. S. Petrović, A dilation theory for polynomially bounded operators, J. Funct. Anal. 108 (1992), 458-469.

4. Operator Theory 20 (1994), 364-376. 
5. B. Sz.-Nagy, Sur les contractions de l'espace de Hilbert, Acta Sci. Math. (Szeged) 15 (1953), 87-92.

6. B. Sz.-Nagy and C. Foias, Harmonic analysis of operators on Hilbert space, North-Holland, Amsterdam, 1970.

DEPARTMENT OF MATHEMATICS, INDIANA UNIVERSity, BloOMington, INDIANA 47405

E-mail address: bercoviceindiana.edu

E-mail address: petroviceiu-math.math.indiana.edu 